\title{
Deprogram and reprogram to solve the riddle of insulin resistance
}

\author{
Victoria L. Tokarz, ${ }^{1,2}$ Paul Delgado-Olguín,,$^{3,4,5}$ and Amira Klip $1,2,6,7$ \\ 'Cell Biology Program, The Hospital for Sick Children, Toronto, Canada. ${ }^{2}$ Department of Physiology, University of Toronto, Toronto, Canada. ${ }^{3}$ Translational Medicine Program, The Hospital for Sick Children, \\ Toronto, Canada. ${ }^{4}$ Department of Molecular Cenetics, University of Toronto, Toronto, Canada. ${ }^{5}$ Heart \& Stroke Richard Lewar Center of Excellence, Toronto, Canada. ${ }^{6}$ Department of Biochemistry and \\ 'Department of Paediatrics, University of Toronto, Toronto, Canada.
}

\begin{abstract}
Skeletal muscle preeminently determines whole-body glycemia. However, the molecular basis and inheritable influence that drive the progression of insulin resistance to type 2 diabetes remain debated. In this issue of the $J C l$, Haider and Lebastchi report on their use of induced pluripotent stem cell-derived (iPSC-derived) myoblasts (iMyos) to uncover multiple phosphoproteomic changes that carried over from the human to the cellculture system. In this system devoid of in vivo influences, the researchers annotated changes between the sexes and between the most and least insulin-sensitive quintiles of a healthy population (defined by steady-state blood glucose levels). Many phosphoproteomic differences were detected in the absence of insulin, revealing that changes in the basal landscape of cells determine the efficiency of insulin action. Basal and insulin-dependent deficiencies of iPSCs and iMyos likely involve genetic and epigenetic determinants that modulate insulin sensitivity.
\end{abstract}

\section{Deconstruct to conquer insulin resistance}

Skeletal muscle glucose uptake is crucial for the maintenance of glycemia and for providing a carbon source to this highly energy-demanding tissue (1). Muscle fibers (multinucleated, 3D cell arrays) remove dietary glucose from blood in response to insulin. Therefore, insulin resistance, manifested primarily as reduced muscle glucose uptake, has been a long-standing health concern. Moreover, with insulin resistance being a key predictor and driver of type 2 diabetes (T2D), it is paramount to dissect the molecular underpinnings of this condition. Understandably, major research efforts focus more on how insulin acts in health and disease (i.e., the insulin-signaling cascade) and less on whether conditions prevailing in the muscle may determine insulin function. Accordingly, a plethora of studies have examined how environmental and behavioral factors (obesity, diet composition, energy balance) influence insulin action in vivo or in muscle ex vivo. In parallel, studies in primary myotubes or muscle cell lines have typically explored the effects of fats, cytokines, or stress factors on insulin action. Those in vivo and in vitro models therefore assume that insulin resistance arises (or is uncovered) by environmental cues. Yet studies over the years find that primary myotubes in culture derived from muscles of T2D-afflicted individuals retain defects in glucose uptake, metabolism, and insulin-derived signals compared with those from healthy controls in the absence of factors circulating in vivo (2-6). This observation harnesses the concept that cell conditions carry over from the organ-

\section{Related Article: https://doi.org/10.1172/JCI151818}

Conflict of interest: The authors have declared that no conflict of interest exists.

ism to the cell culture. Moreover, the recognized familial risk for T2D suggests that hereditary determinants predispose to the development of insulin resistance. In the absence of specific mutations defining insulin resistance in the rank and file T2D, detangling the molecular basis of these conditions has proven challenging.

In this issue of the JCI, Haider and Lebastchi et al. (7) use a diabetes-in-a-dish approach to explore the molecular origins of skeletal muscle insulin resistance. This model deprograms fully differentiated cells to yield induced pluripotent stem cells (iPSCs), followed by reprogramming these into skeletal muscle myoblasts, to generate a plentiful source of skeletal muscle cells. When iPSC-derived myoblasts (iMyos) were cultured in vitro, they replicated key features of their insulin-sensitive or insulin-resistant donors, such as reduced glucose uptake and impaired activation of the proximal insulin-signaling cascade. This strategy is rooted in the authors' investigations in iMyos stemming from individuals with insulin receptor mutations (8) or with T2D (9). Global phosphoproteomic analysis revealed cell-inherent differences in iMyos in both the monogenic disease and T2D.

In Haider and Lebastchi et al., blood-derived iPSCs were generated from a healthy population that spans the normal range of steady-state plasma glucose (SSPG). Despite examining iMyos from a relatively small number of individuals, the analysis revealed marked differences in the phosphoprotein landscape and functional outcomes between the extreme quintiles of SSPG. Multiple differences were observed before and after insulin administration, highlighting basal state alterations that track with the least insulin-sensitive phenotypes within the normal range of SSPG. Moreover, the study allowed the authors to identify networks of dysregulated phosphorylation. Strikingly, the phosphoproteomic signatures were 


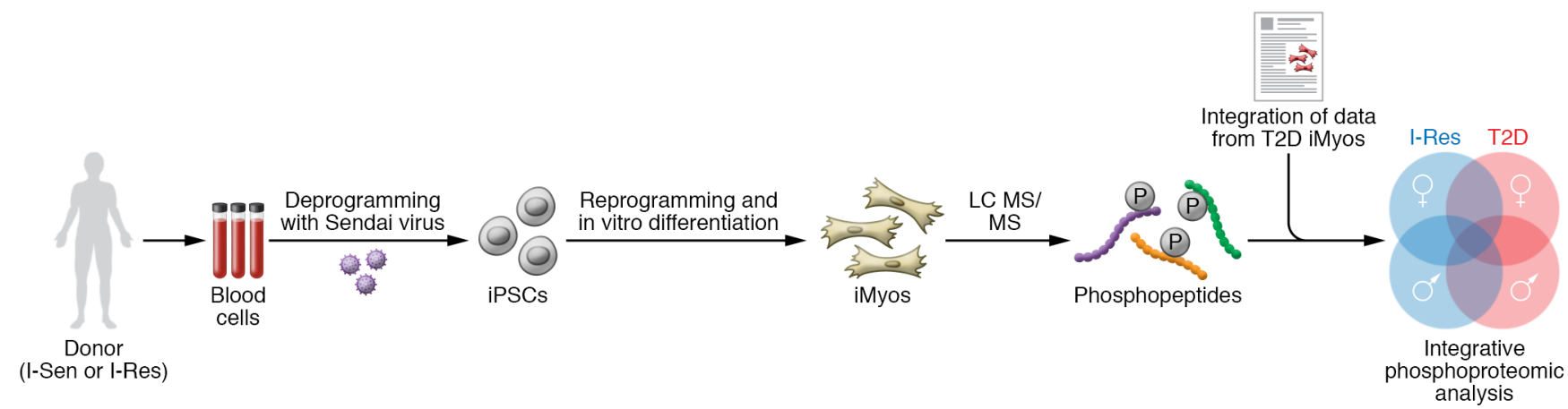

Figure 1. Diabetes-in-a-dish approach to exploring the molecular origins of skeletal muscle insulin resistance. Blood cells from I-Sen or I-Res donors (men and women) were deprogrammed with Sendai virus to generate iPSCs, then redifferentiated in vitro into iMyos. The unique phosphoproteomic signature of these iMyos (before or after insulin stimulation) was compared with that of iMyos from T2D donors to reveal overlapping dysregulated phosphorylation networks in I-Res and T2D iMyos and a sex-specific phosphoproteomic fingerprint. I-Res and T2D iMyos exhibited dysregulated phosphorylation and signaling networks in I-Res and T2D iMyos, including Rho/Rac GTPase signaling, chromatin organization, and RNA splicing and processing. These phosphoproteomic changes were reflected in functional differences in the DNA damage response and actin cytoskeleton remodeling. Notably, I-Sen and I-Res iMyos displayed a sex-specific phosphoproteomic fingerprint. The dysregulated phosphoproteome in I-Sen and I-Res iMyos under basal conditions implies a cell-autonomous defect in insulin handling. Genetic and epigenetic mechanisms of inheritability that could lead to an altered phosphoproteome and downstream functional outcomes warrant further investigation. Overall, the work of Haider and Lebastchi et al. (7) represents a major leap in our understanding of insulin resistance. LC MS/MS, liquid chromatography-tandem mass spectrometry.

sexually dimorphic. Notably, a subset of the phosphoprotein changes in the highest SSPG quintile (suggesting most insulin resistant) also overlapped with changes that this research group recently found in iMyos of T2D individuals (9). This observation resonates with Tirosh et al.'s report (10) showing that the highest quintile blood glucose levels in 13,163 healthy individuals tracked prospectively with a higher risk of developing T2D. The findings by Haider and Lebastchi et al. (7) take us one step closer toward defining determinants of cell-autonomous muscle insulin resistance by homing in mechanistically on the concept that muscle-intrinsic properties conveying this susceptibility are genetically determined.

Mechanistically, the most salient finding of this study is that broad phosphorylation networks in Rho/Rac GTPase signaling, chromatin organization, and RNA splicing and processing were dysregulated in both insulin-resistant (I-Res) and T2D iMyos (7) relative to insulin-sensitive (I-Sen) iMyos. This global phosphoproteomic signature reveals strong, sex-specific fingerprints that segregate along or away from the degree of insulin sensitivity (Figure 1). Functional testing of some of the phosphoproteomic changes uncovered differences in the DNA damage response (defined by apurinic/apyrimidinic sites) and in actin cytoskeleton remodeling (measured by RhoA activation). Impor- tantly, these complex changes segregated with reduced insulin-dependent glucose uptake in I-Res iMyos compared with I-Sen iMyos, and insulin-induced actin remodeling indeed contributes to insulin-stimulated GLUT4 translocation (1).

\section{Genetics and beyond}

Beyond the wide-angle approach of uncovering differences in the phosphoproteome and some of its associated signaling, metabolic, or structure/repair pathways, it is essential to explore the origin for the persistence of these changes. Could the changes in the phosphoproteome that are recapitulated in iMyos arise from somatic mutations occurring in vivo? If so, the origin of iPSCs may be important. While Batista et al. (9) generated iMyos from muscle-derived iPSCs, Haider and Lebastchi et al. (7) generated iPSCs from blood cells. Given that there may be tissuespecific developmental cues retained in iPSCs (11), it will become important to compare the phosphoproteome of iMyos derived from blood and muscle iPSCs as well as of adipocytes and iMyos generated from the same iPSCs.

The structure and accessibility of chromatin influence the cellular plasticity required for reprogramming gene expression in somatic cells and reestablishing the pluripotent state in iPSCs (12). The findings of Haider and Lebastchi et al. are compatible with the possibility that a donor's genetic makeup cooperates with epigenetic mechanisms to control insulin sensitivity as a cell-autonomous response to insulin stimulation in iMyos. DNA methylation marks and miRNAs that persist in iPSCs are plausible mediators of an acquired signature. DNA methylation marks that are deposited during establishment of cell identity are erased during reprogramming $(13,14)$. However, donor-specific methylation marks are retained in iPSCs. Similarly, expression of a subset of miRNAs, including members of the Let-7 family, is conserved in iPSCs derived from blood progenitors (15). Notably, Let-7a is elevated in primary myotubes of T2D individuals in the absence of the circulating environment that the muscle would have faced in vivo (16). Therefore, residual epigenetic marks or miRNAs might contribute to a transcriptional program that controls insulin sensitivity.

Altering phosphorylation of histone modifiers could further affect the transcriptional response of iMyos to insulin, for example, in states of stress or in disease. Haider and Lebastchi et al. (7) revealed that epigenetic regulators influencing histone modification, chromatin accessibility and organization, miRNA processing, and maintenance of DNA methylation, among others, were differentially phosphorylated in I-Sen and I-Res iMyos, and vast correlates were found between iMyos from I-Res subjects and 
those with overt diabetes $(7,9)$. It will be interesting to explore whether the phosphorylation changes found in Haider and Lebastchi et al. (7) affect the function of epigenetic regulatory proteins. Crossreferencing genome-wide distribution of DNA methylation and key histone marks with gene expression in somatic cells and iMyos could address this possibility.

Finally, epigenetic modifiers require intermediates of metabolic pathways as cofactors (17). The metabolic profile is also reprogrammed during reestablishment of pluripotency $(18,19)$. In addition to epigenetic marks, metabolomic characteristics from the cell of origin are also retained in iPSCs. Since iPSCs derived from fibroblasts utilize carbohydrates and amino acids differently than pluripotent embryonic stem cells in response to oxygen (20), the influence of metabolites retained in I-Sen and I-Res iPSCs may differ. Hence, metabolites associated with insulin resistance in donors may also influence the function of epigenetic modifiers as potential regulators of the cell-autonomous response to insulin in iMyos. In aggregate, the results by Haider and Lebastchi et al. (7) lay the foundation for investigating valuable aspects of the transcriptional and epigenetic control of insulin resistance (Figure 1).

\section{The reconstruction}

The changes persisting after the deprogram-reprogram cycle that takes place upon the respective generation of iPSCs and iMyos not only reflect a state of susceptibility to insulin resistance upon an environmental challenge, but also reveal an inherent cellular insulin resistance. When does an individual cross the threshold from mildly insulin resistant within the normal range of SSPG to full-fledged insulin resistant, as defined in the metabolic syndrome? Do triggering conditions such as lipids and obesity remove a protective mechanism? Or do they further aggravate insulin resistance?

The authors identify phosphorylation networks dysregulated by insulin resistance, raising the question of which are drivers of insulin resistance (those that directly confer insulin resistance) and which are passengers (those that occur because of, but do not drive, insulin resistance)? How do and which specific changes in the phosphoproteome cause the observed defects in signaling and glucose uptake? Toward identifying drivers, prospective studies could characterize the phosphoproteomic signature of iMyos from insulin-resistant individuals at different time points as the cell donors progress (or not) toward the development of T2D.

The manipulability of the diabetesin-a-dish model developed by Haider and Lebastchi et al (7) is a substantial advance. Adaptation of the system to study terminally differentiated cells of other insulinresponsive tissues (such as adipocytes) could identify phosphoproteomic signatures that are tissue specific and those that are preserved across tissues. Similarly, studying iPSC-derived myocytes from patients with other metabolic diseases may reveal a shared origin. Along with the highly valuable phosphoprotein annotation, future examinations of proteomics, protein subcellular localization, and complex formation will shed further light on the mechanistic basis of insulin resistance.

\section{Acknowledgments}

VLT is supported by a Doctoral Studentship from the Canadian Institutes of Health Research (CIHR). PDO is supported by the CIHR (162208 and 149046), the Heart and Stroke Foundation of Canada (G-170018613), and the Natural Sciences and Engineering Research Council of Canada (NSERC) (500865). AK's work is supported by foundation grant FDN-143203 from the CIHR. We thank Anna Krook and Scott Frendo-Cumbo for helpful discussions.

Address correspondence to: Amira Klip, Cell Biology Program, Research Institute, The Hospital for Sick Children, 686 Bay Street Room 19.9-709, Toronto, Ontario, M5G 0A4, Canada. Phone: 416.813.6392; Email: amira@sickkids.ca. Or to: Paul Delgado-Olguín, Translational Medicine Program, The Hospital for Sick Children, 686 Bay Street, Room 10.9715, Toronto, Ontario, M5G 0A4, Canada. Phone: 416. 813.5080; Email: paul.delgadoolguin@ sickkids.ca.

1. Sylow L, et al. The many actions of insulin in skeletal muscle, the paramount tissue determining glycemia. Cell Metab. 2021;33(4):758-780.

2. Gaster M. The diabetic phenotype is preserved in myotubes established from type 2 diabetic subjects: a critical appraisal. APMIS. 2019;127(1):3-26.
3. Gaster M, et al. The diabetic phenotype is conserved in myotubes established from diabetic subjects: evidence for primary defects in glucose transport and glycogen synthase activity. Diabetes. 2002;51(4):921-927.

4. Al-Khalili L, et al. Profiling of human myotubes reveals an intrinsic proteomic signature associated with type 2 diabetes. Transl Proteomics. 2014;2(1):25-38.

5. Bakke SS, et al. Myotubes from severely obese type 2 diabetic subjects accumulate less lipids and show higher lipolytic rate than myotubes from severely obese non-diabetic subjects. PLoS One. 2015;10(3):e0119556.

6. Ciaraldi TP, et al. Altered myokine secretion is an intrinsic property of skeletal muscle in type 2 diabetes. PLoS One. 2016;11(7):e0158209.

7. Haider N, et al. Signaling defects associated with insulin resistance in nondiabetic and diabetic individuals and modification by sex. J Clin Invest. 2021;131(21): 151818.

8. Iovino S, et al. Myotubes derived from human-induced pluripotent stem cells mirror in vivo insulin resistance. Proc Natl Acad Sci U S A. 2016;113(7):1889-1894.

9. Batista TM, et al. A cell-autonomous signature of dysregulated protein phosphorylation underlies muscle insulin resistance in type 2 diabetes. Cell Metab. 2020;32(5):844-859.

10. Tirosh A, et al. Normal fasting plasma glucose levels and type 2 diabetes in young men. $N$ Engl J Med. 2009;353(14):1454-1462.

11. Quattrocelli M, et al. Mesodermal iPSCderived progenitor cells functionally regenerate cardiac and skeletal muscle. J Clin Invest. 2015;125(12):4463-4482.

12. Nashun B, et al. Reprogramming of cell fate: epigenetic memory and the erasure of memories past. EMBO J. 2015;34(10):1296-1308.

13. Koche RP, et al. Reprogramming factor expression initiates widespread targeted chromatin remodeling. Cell Stem Cell. 2011;8(1):96-105.

14. Maherali N, et al. Directly reprogrammed fibroblasts show global epigenetic remodeling and widespread tissue contribution. Cell Stem Cell. 2007;1(1):55-70.

15. Vitaloni M, et al. MicroRNAs contribute to induced pluripotent stem cell somatic donor memory. J Biol Chem. 2014;289(4):2084-2098.

16. Jiang LQ, et al. Autocrine role of interleukin-13 on skeletal muscle glucose metabolism in type 2 diabetic patients involves microRNA let-7. Am JPhysiol Endocrinol Metab. 2013;305(11):1359-1366.

17. Boon R, et al. Nuclear metabolism and the regulation of the epigenome. Nat Metab. 2020;2(11):1190-1203.

18. Panopoulos AD, et al. The metabolome of induced pluripotent stem cells reveals metabolic changes occurring in somatic cell reprogramming. Cell Res. 2012;22(1):168-177.

19. Prigione A, et al. The senescence-related mitochondrial/oxidative stress pathway is repressed in human induced pluripotent stem cells. Stem Cells. 2010;28(4):721-733.

20. Harvey AJ, et al. Physiological oxygen culture reveals retention of metabolic memory in human induced pluripotent stem cells. PLoS One. 2018;13(3):e0193949. 